\title{
Preface
}

\section{Body Contouring After Massive Weight Loss}

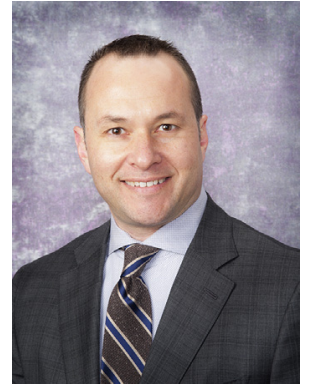

Jeffrey A. Gusenoff, MD Editor
Body contouring after weight loss has become a growing niche in plastic surgery. Over the past decade, the rise in worldwide obesity has led to evolving trends in bariatric surgery that have a direct impact on the practice of plastic surgery. From banding procedures, to stomach plication, balloons, and sleeve procedures, there is a constant array of surgical and nonsurgical treatments aimed to combat the obesity epidemic. A major pitfall of massive weight loss is the significant skin relaxation that occurs afterwards. The excess skin makes patients feel uncomfortable, unhygienic, and in need for further improvement. Some even feel worse with the loose skin than when they were heavy. Patients then turn to plastic surgery to help complete the process of their weight loss journey with innovative procedures done in the safest manner.

The different procedures offered for body contouring after weight loss are numerous and include challenging operations like lower body lifts, back lifts, and thigh lifts that pose significant challenges. Additional areas that require new techniques or advances include the breasts, arms, face, and neck.

This issue of Clinics in Plastic Surgery covers many key areas of the subspecialty, including a review of the scope of management of the obesity epidemic, patient evaluation prior to body contouring with an emphasis on patient safety, how to stage patients appropriately, and how to perform state-of-the-art techniques by world leaders in the field. This issue also covers important topics of pain control in body contouring procedures as well as a comprehensive understanding of complications that can arise and how to manage or avoid them.

I am very appreciative of the work of all the contributors for this issue; without their efforts and dedication, this collection would not be possible. It is wonderful to have such excellent minds and technically gifted surgeons in the field of body contouring, constantly working to evolve the field and improve outcomes for this special population. I hope this international collection of authors provides a well-rounded review of the field of body contouring today, provides insight into the challenges of body contouring, and opens the door to future innovations in the field.

Jeffrey A. Gusenoff, MD University of Pittsburgh 3380 Boulevard of the Allies, Suite 180 Pittsburgh, PA 15213, USA

E-mail address: gusenoffja@upmc.edu 